\title{
PROCESSING-AWARE COMPRESSION FOR SENSOR NETWORKS
}

\author{
Lavanya Vasudevan and Antonio Ortega \\ Department of Electrical Engineering \\ Signal and Image Processing Institute \\ Integrated Media Systems Center \\ University of Southern California, \\ Los Angeles, California 90089-2564. \\ \{vasudeva, ortega\}@sipi.usc.edu
}

\begin{abstract}
In sensor networks, where power and bandwidth are at a premium, there is a clear need to use compression to limit the amount of information exchanged by the sensors. In this paper we study the signal compression problem in situations where signals are being processed for the purpose of source localization. In these scenarios compression should be optimized for the accuracy of source localization, rather than to provide a reproduction of the signals with some desired fidelity. We show how this leads to novel design techniques that have clear advantages over standard quantizer design approaches.
\end{abstract}

\section{INTRODUCTION}

Networks of sensing nodes have excited much interest in recent literature. Typically, large numbers of these sensors are randomly deployed, and coordinate to establish a communication network [1], [2]. Noisy signal measurements are collected from each sensor, and fused at a central processor to estimate some environmental parameter. For example, in a source tracking application, different sensor observations are correlated to estimate the relative time delays between them, and thence, the source location [3]. While each sensor has both processing and communication elements, limitations on power and bandwidth motivate the use of compression at the sensor - the rule of thumb is that computation is cheaper than communication. Compression techniques would exploit the high degree of both temporal and spatial correlation of sensor data.

Recent work on distributed source coding [4] has leveraged spatial redundancy of sensor data for compression. The fidelity criterion in these methods is, as with most traditional quantization techniques, the error between the original and the reconstructed observations. However, the real objective

This work has been supported in part by NASA under grant AIST0122-0005 in many sensor processing scenarios is to estimate some signal parameter from the quantized and fused observations. Given the fusion algorithm at the center, each sensor should quantize its data so as to preserve the quality of the ultimate objective. In the tracking application described above, the fidelity criterion would be the degradation in the final source location estimate, rather than in the sensor data itself. Therefore, the goal for an application specific encoder in this context would be to achieve the best estimate at a given rate, or minimize the rate required to achieve a location estimate with given accuracy.

In this paper, we investigate whether special quantizer design techniques are required for this scenario. We propose and test quantizer designs where the goal is to optimize the accuracy of the location estimate.

\section{PROBLEM STATEMENT}

We consider the case of two sensors, each capturing and transmitting a delayed and noisy version of the same signal. The task is to estimate the time delay $\delta$ between the received signals.

$$
\begin{aligned}
& x_{1}(m)=x(m)+w_{1}(m) \\
& x_{2}(m)=x(m-\delta)+w_{2}(m)
\end{aligned}
$$

The time delay between $x_{1}(m)$ and $x_{2}(m)$ is calculated from the cross-correlation function between the signals; Our best estimate of $\delta$ would simply be the lag at which the cross-correlation peaks. Now, if we were to quantize the two signals,

$$
\begin{aligned}
& \hat{x}_{1}(m)=x_{1}(m)+e_{1}(m) \\
& \hat{x}_{2}(m)=x_{2}(m)+e_{2}(m)
\end{aligned}
$$


where $e_{1}(m)$ and $e_{2}(m)$ are the quantization noise values. The new (empirical) cross-correlation function, based on $M$ input samples, is

$$
\hat{R}_{M}(n)=\frac{1}{M} \sum_{m} \hat{x}_{1}(n+m) \hat{x}_{2}(m)
$$

While conventional encoding schemes seek to minimize the squared error between $\hat{x}_{i}$ and $x_{i}$, the new scheme seeks quantizers such that $\hat{R}_{M}(n)$ still peaks at $n=-\delta$.

\section{QUANTIZER FORMULATION}

Without loss of generality, we assume that the true delay between the signals is zero. Then,
At high rates, one can think of standard approximations used in quantizer analysis which assume that the quantization noise $e_{q}$ is white and uncorrelated with the input signal. Under those conditions, the expected value of $e_{R_{M}}(n)$ at $n=0$ will tend to the power of the noise, whereas the expected value of the error at other lags would be zero. The error at lag zero is then higher, on average, than the error at any other lag. This is not necessarily true at lower quantization rates, and an optimal quantizer design would have to aim at minimizing the error in correlation at any lag. However, as a first approximation, we concentrate on minimizing the error at lag zero:

$$
e_{R_{M}}(0)=\frac{1}{M} \sum_{m}\left(\hat{x}^{2}(m)-x^{2}(m)\right) .
$$

Writing the total error as the sum of the errors in each $R_{M}(n)=\frac{1}{M} \sum_{m}\left(x(m)+w_{1}(m)\right)\left(x(m+n)+w_{2}(m+n)\right.$ guantization bin $Q_{i}$, we have

and

$\hat{R}_{M}(n)=\frac{1}{M} \sum_{m}\left(\hat{x}(m)+\hat{w}_{1}(m)\right)\left(\hat{x}(m+n)+\hat{w}_{2}(m+n)\right)$

$$
\begin{array}{r}
\frac{1}{M} \sum_{i}\left(\sum_{m: x(m) \in Q_{i}} \hat{x}^{2}(m)-x^{2}(m)\right) \\
=\frac{1}{M} \sum_{i} M_{i}\left(\hat{x}_{i}^{2}-\frac{\sum_{M_{i}} x^{2}(m)}{M_{i}}\right)
\end{array}
$$

Under the assumption that the noise affecting each of the sensors is white, and that the two noise sources are uncorrelated, the effect of the noise will be eliminated if the correlation is computed over a sufficient number of samples $M$ :

$$
R_{M}(n) \approx \frac{1}{M} \sum_{m} x(m) x(m+n)
$$

and

$$
\hat{R}_{M}(n) \approx \frac{1}{M} \sum_{m} \hat{x}(m) \hat{x}(m+n)
$$

Thus, when we consider the noiseless case, our goal is to design a quantizer such that given a signal $x(m)$, its quantized version $\hat{x}(m)$ leads to empirical autocorrelations that are as close as possible to those that would have been computed based on the original signal. When noise power is unknown, the best we can do is use the quantizer optimized for the noiseless case.

At any lag $n$, the error in the correlation function can be written as follows.

$$
\begin{array}{r}
e_{R_{M}}(n)=\hat{R}_{M}(n)-R_{M}(n) \\
=\frac{1}{M} \sum_{m}(\hat{x}(n+m) \hat{x}(m)-x(m+n) x(m)) \\
=\frac{1}{M} \sum_{m}\left(e_{q}(n+m) e_{q}(m)-2 x(m+n) e_{q}(m)\right)
\end{array}
$$

where $M_{i}$ is the number of input samples in quantization bin $Q_{i}$. Now,

$$
E\left[e_{R_{M}}(0)\right]=\frac{1}{M} \sum_{i} M_{i}\left(\hat{x}_{i}^{2}-\frac{1}{M_{i}} \sum_{M_{i}} E\left[x^{2} / x \in Q_{i}\right]\right)
$$

which can be made zero simply by setting

$$
\hat{x}_{i}^{2}=E\left[x^{2} / x \in Q_{i}\right]
$$

Indeed, the new quantizer design is analogous to traditional MSE, which preserves the fidelity in $x$ by minimizing $E\left[(\hat{x}-x)^{2}\right]$; here, we preserve the fidelity in $x^{2}$ (or the correlation at lag zero) by minimizing $E\left[\left(\hat{x}^{2}-x^{2}\right)^{2}\right]$.

Following the analogy with conventional nearest neighbor design, and replacing $x$ by $x^{2}$, we have

$$
x_{p_{i}}^{2}=\frac{\hat{x}_{i}^{2}+\hat{x}_{i+1}^{2}}{2}
$$

where $x_{p_{i}}$ is the partition between adjacent reconstruction levels $\hat{x}_{i}$ and $\hat{x}_{i+1}$.

\section{DISCUSSION}

While the new quantizer design does minimize the error $e_{R}(0)$, the correlation error at other lags also needs to be minimized for a fully optimal design. From Eq.1, $e_{R}(n)$ 
can again be written as the sum of errors in each quantization bin:

$$
\begin{aligned}
& \frac{1}{M} \sum_{i} \sum_{m: x(m) \in Q_{i}}(x(m+n) x(m)-\hat{x}(m+n) \hat{x}(m)) \\
& =\frac{1}{M} \sum_{i} \sum_{m: x(m) \in Q_{i}}\left(x(m+n) x(m)-\hat{x}(m+n) \hat{x}_{i}\right)
\end{aligned}
$$

$=\frac{1}{M} \sum_{i} \sum_{m: x(m) \in Q_{i}}\left(x(m+n) x(m)-\left(x(m+n)+e_{q}(m+n)\right) \hat{x}_{i}\right)$

$=\frac{1}{M} \sum_{i} \sum_{m: x(m) \in Q_{i}}\left(x(m+n)\left(x(m)-\hat{x}_{i}\right)+e_{q}(m+n) \hat{x}_{i}\right)$

For a good quantization scheme, especially with symmetric reconstruction levels, the average quantization error goes to zero; Hence, at $n \neq 0$, the second term in Eq.12 may be ignored for large $M$. For IID sources, the first term in the summation is minimized when $\hat{x}_{i}=E\left[x / x \in Q_{i}\right]$ - which is in fact the traditional MSE centroid. In general, minimizing the error at lag zero does not guarantee minimizing the error at other lags, and there is a trade-off between the two. In this study, we present results optimized for $e_{R}(0)$, ignoring (and possibly incurring a penalty due to) $e_{R}(n)$.

\section{EMPIRICAL METHOD}

Given a training set, sequence length $M$, and the required number of quantization levels, we use the standard approach of alternating two steps, namely, fixing the encoder and optimizing the decoder, and then fixing the decoder and optimizing the encoder.

Focusing on the scalar quantization case, the design is as follows.

In the first step, we assume that the quantization bins have been chosen and then we select the reproduction level for each bin according to Eq. 7. In the second step, we assume that the reproduction levels are fixed, and we decide how to assign an input in the training set to a given reproduction level with the target to minimize the error variance. Partitions between adjacent quantization bins evolve as Eq. 8.

\section{PRELIMINARY RESULTS}

Computer simulations were run with randomly generated source signals, with both IID Gaussian and IID Uniform distributions. The additive noise at each sensor was simulated as white Gaussian. Scalar quantization codebooks and partitions were iteratively generated (e.g., Table 1) for the conventional MSE quantizer (denoted MSE) and the new quantizer (denoted NP-NC) derived above. As a comparison case, we also experimented with a halfway-optimal quantizer that combined the new centroid condition, with standard nearest neighbor partitioning (denoted MSE-NC). Received signals at both sensors, with true delay between them set to zero, were quantized with each of the three schemes. The average values of the index at which the correlation function peaked was measured. These peak indices were compared with the unquantized peak estimates for each of the quantizers at several quantization rates, noise levels and sequence lengths (Figs. 1, 2, 3, 4). Average error values, for both quantization error $e_{q}$ and correlation error $e_{R}(0)$, were also compared for the different quantizers (e.g., Table 2).

We see that in all cases, estimates of the correlation peak do improve as sequence length increases, since the additive sensor noise tends to average out. At shorter sequence lengths, the quantization schemes with the new centroid alone (MSE-NC) and new partition with the new centroid (NP-NC) give estimates that are closer to the true peak at 0 , than MSE. Table 2 shows that while MSE may minimize the variance of the quantization error $\sigma_{e_{q}}^{2}, \mathrm{NP}-\mathrm{NC}$ is indeed the best choice to minimize the mean and variance of the correlation error at lag zero. Further, the distribution of quantization bin probabilities (Table 3) suggests that we may be able to achieve a lower entropy with the new quantization scheme than with MSE.

In most cases, the new scheme does present an improvement over standard quantization, but the new estimate is not as close to the true correlation peak as might be desired; and in some cases (Fig.4), the new scheme is not clearly better. The explanation for this might be looked for in Section 4; the quantizer design optimized for $e_{R}(0)$ does incur a penalty from the correlation error at other lags. The question of a fully optimal quantizer design for this problem remains open.

\section{REFERENCES}

[1] D. Estrin, R. Govindan, J. Heidemann and S. Kumar, "Next century challenges: Scalable coordination in sensor networks," in Mobicom 99, ACM, Aug. 1999.

[2] J. Agre, L. Clare, "An integrated architecture for cooperative sensing networks," in IEEE Computer, vol. 33, no. 5, May 2000.

[3] J. C. Chen, Kung Yao and R. E. Hudson, "Source localization and beamforming," in IEEE Signal Processing Magazine, vol. 19, no. 2, March 2002, pp. 30-39.

[4] S. S. Pradhan, J. Kusuma and K. Ramchandran, "Distributed compression in a dense microsensor network," in IEEE Signal Processing Magazine, vol. 19, no. 2, March 2002, pp. 51-60. 


\begin{tabular}{|c|c|c|c|c|}
\hline Quantization & $x_{p_{1}}$ & $x_{p_{2}}$ & $x_{p_{3}}$ & \\
\hline$\overline{\mathrm{MSE}}$ & -0.9825 & $\overline{0.0}$ & 0.9825 & \\
\hline MSE-NC & -1.16 & 0.0 & 1.16 & \\
\hline NP-NC & -1.4804 & 0.0 & 1.4804 & \\
\hline Quantization & $\hat{x}_{1}$ & $\hat{x}_{2}$ & $\hat{x}_{3}$ & $\hat{x}_{4}$ \\
\hline$\overline{\mathrm{MSE}}$ & -1.512 & $\begin{array}{c}-0.4530 \\
\end{array}$ & $\overline{0.4530}$ & 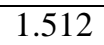 \\
\hline MSE-NC & -1.709 & -0.6115 & 0.6115 & 1.709 \\
\hline NP-NC & -1.960 & -0.736 & 0.736 & 1.960 \\
\hline
\end{tabular}

Table 1. Partitions and Codebooks, Input $N(0,1), 4$ Levels

\begin{tabular}{|c|c|c|c|}
\hline & MSE & MSE-NC & NP-NC \\
\hline \hline$E\left[e_{q}\right], 10^{-3} *$ & -0.4555 & -0.3611 & -0.2551 \\
$\sigma_{e_{q}}^{2}$ & 0.1174 & 0.1311 & 0.1723 \\
$E\left[e_{R}(0)\right]$ & -0.1167 & 0.0009 & 0.0005 \\
$\sigma_{e_{R}}^{2}(0)$ & 0.9371 & 0.7967 & 0.6950 \\
\hline
\end{tabular}

Table 2. Average Error Values, Input $N(0,1), 4$ Levels

\begin{tabular}{|c|c|c|c|c|}
\hline MSE & 0.1633 & 0.3371 & 0.3367 & 0.1629 \\
MSE-NC & 0.1233 & 0.3771 & 0.3765 & 0.1231 \\
NP-NC & 0.0696 & 0.4308 & 0.4300 & 0.0696 \\
\hline
\end{tabular}

Table 3. Bin Probabilities, Input $N(0,1), 4$ Levels

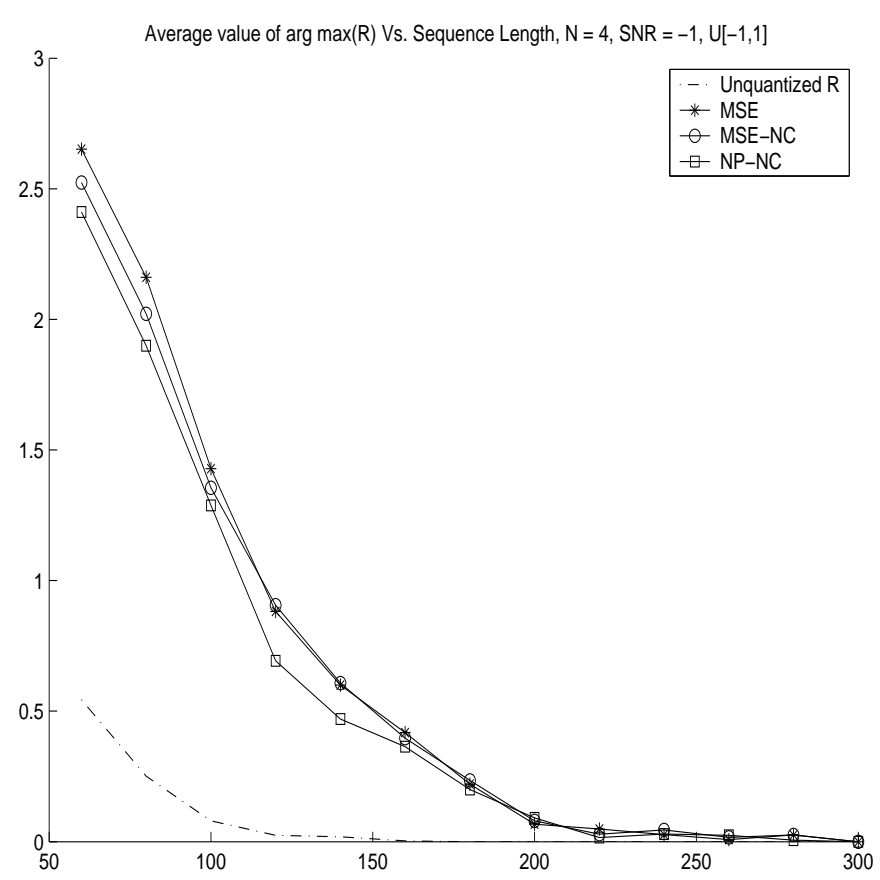

Fig. 1. Uniform Process, $[-1,1],|\arg \max R(n)|$ at $-1 \mathrm{~dB}$ SNR, 4-level Quantization

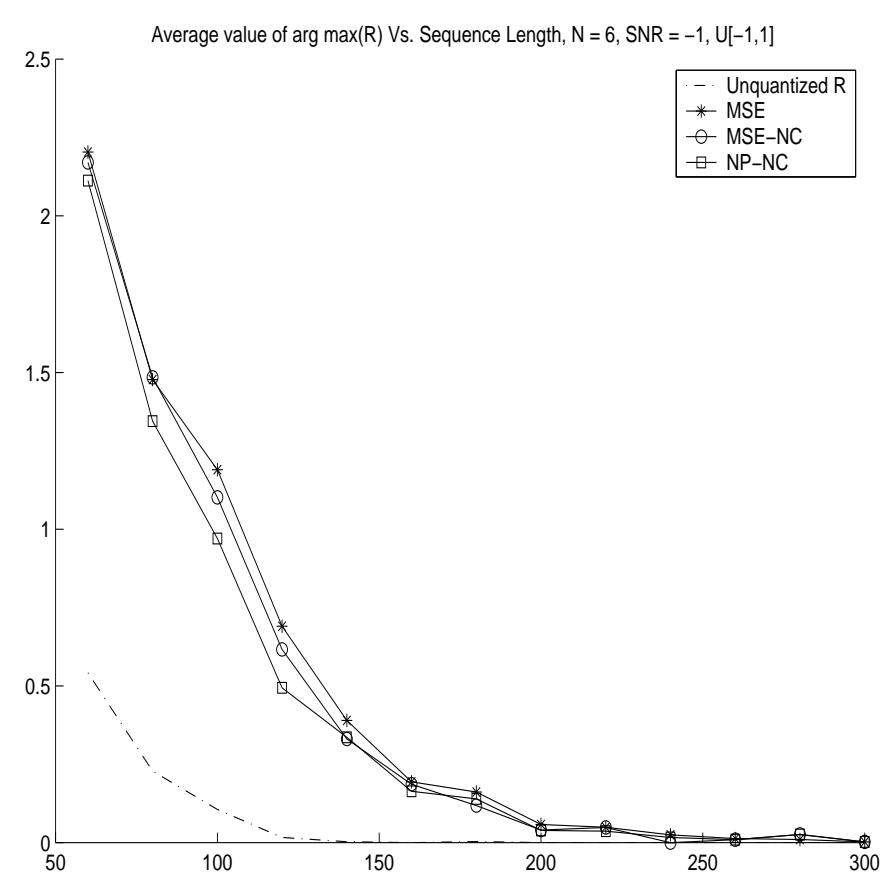

Fig. 2. Uniform Process, $[-1,1],|\arg \max R(n)|$ at $-1 \mathrm{~dB}$ SNR, 6-level Quantization

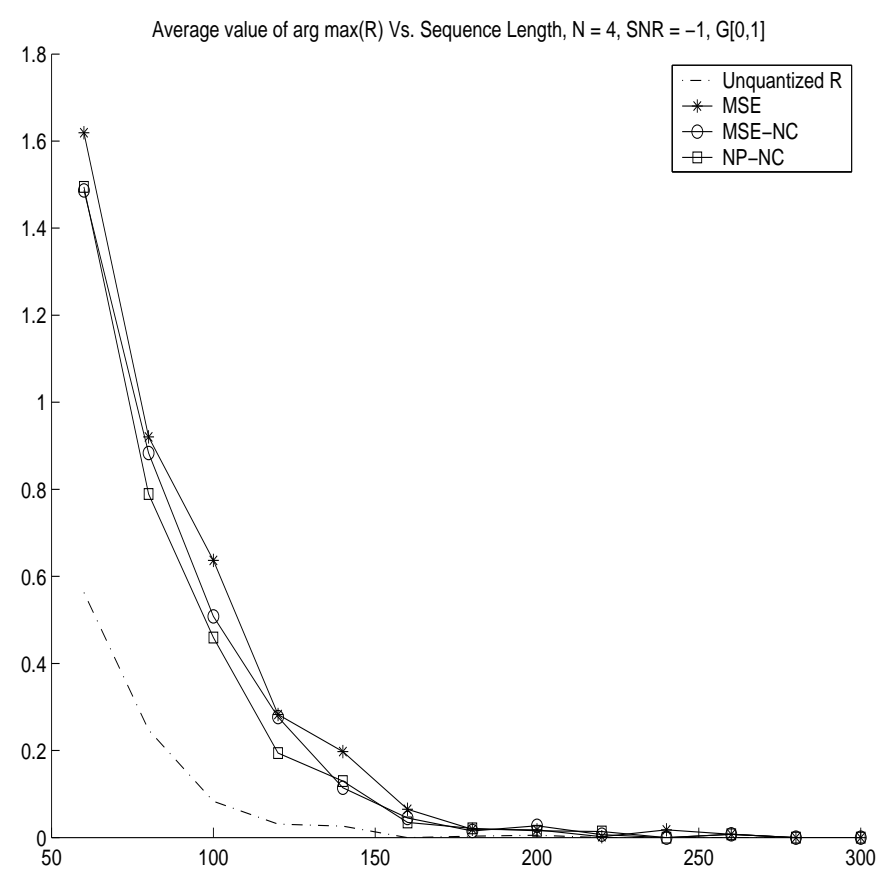

Fig. 3. Gaussian Process, $N(0,1)$, $|\arg \max R(n)|$ at $-1 \mathrm{~dB}$ SNR, 4-level Quantization 


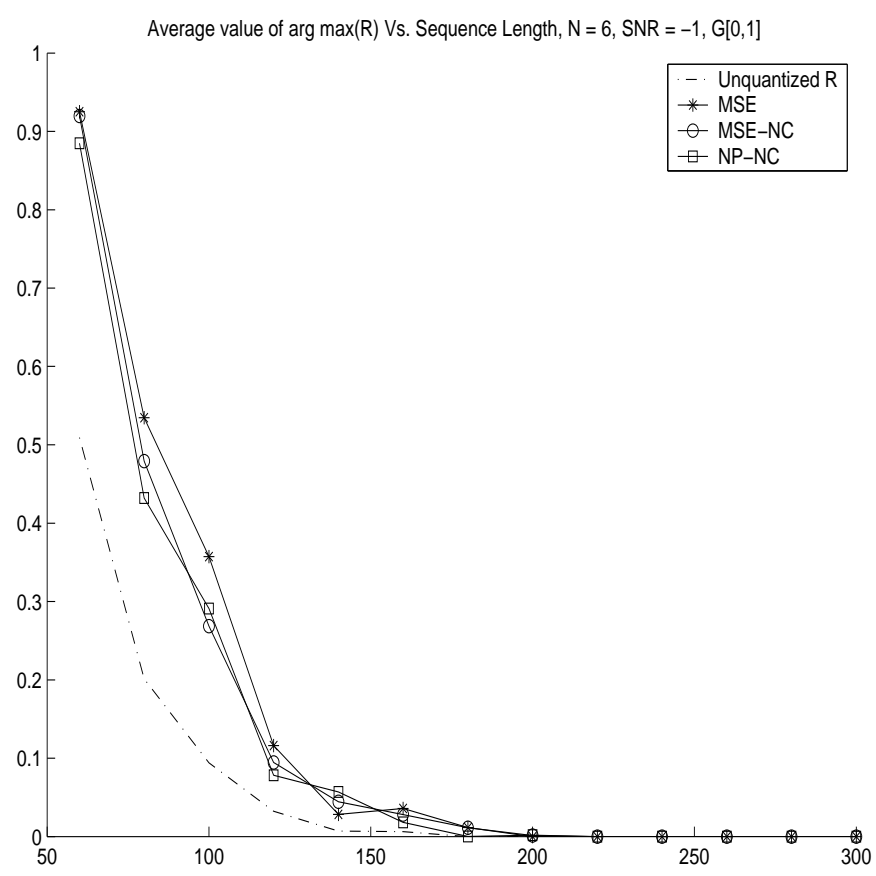

Fig. 4. Gaussian Process, $N(0,1),|\arg \max R(n)|$ at $-1 \mathrm{~dB}$ SNR, 6-level Quantization 